\title{
Explotación y distribución de la captura artesanal de camarón en el Sistema Lagunar Huave, Oaxaca, México
}

\section{Exploitation and distribution of artisanal shrimp catch in the Huave Lagoon System, Oaxaca, Mexico}

\author{
Pedro Cervantes-Hernández ${ }^{*}$, Ma. Isabel Gallardo-Berumen ${ }^{1}$ y Saúl J. Serrano-Guzmán ${ }^{2}$
}

\begin{abstract}
RESUMEN
En el Sistema Lagunar Huave, la explotación y la distribución de la captura artesanal de camarón resultaron afectadas por diferencias en la calidad hidrológica (temperatura superficial, salinidad, oxígeno disuelto y turbidez) y la pérdida en la cobertura de manglar. Las mejores condiciones hidrológicas con extensas áreas de manglar fueron observadas en las lagunas Oriental y Occidental, donde el 97\% de la captura artesanal de camarón fue obtenida. Las peores condiciones hidrológicas con escasas áreas de manglar fueron registradas al interior $(0.35 \%)$ y en la entrada $(0.85 \%)$ del Mar Tileme, donde la captura artesanal de camarón fue escasa, registrando una tasa de mortalidad natural de 0.82 mensual. El período analizado fue de 1999 al 2007, pero de acuerdo con los antecedentes bibliográficos, el reclutamiento marino de camarones juveniles en este sistema lagunar comenzó a descender en el Golfo de Tehuantepec, durante los años ochenta. La presión por pesca fue modificada por las condiciones ambientales imperantes en cada sección del cuerpo lagunar, esta fue "muy baja" en el Mar Tileme, "alta" en las lagunas Oriental y Occidental y "baja" en la Laguna Inferior.
\end{abstract}

Palabras claves: Evaluación, distribución, camarón, mangle, lagunas costeras.

\begin{abstract}
In the Huave Lagoon System, the artisanal exploitation and distribution of shrimp were affected by the loss of hydrological quality (surface temperature, salinity, dissolved oxygenate and turbidity) and mangrove cover. The best hydrological conditions with extensive mangrove areas were observed in the Oriental and Occidental Lagoons, where $97 \%$ of artisanal shrimp catches was obtained. The worst hydrological conditions with little mangrove area were recorded within $(0.35 \%)$ and in the entrance $(0.85 \%)$ of the Mar Tileme Lagoon, where artisanal shrimp catches were low, recording a natural mortality rate of 0.80 per month. The period analyzed was from 1999 to 2007; however, according to the literature, the marine recruitment of juvenile shrimp in this lagoon system began to decline in the Gulf of Tehuantepec during 1980s. Fishing pressure was modified by the prevailing environmental conditions in each lagoon section. This pressure was "very low" in the Mar Tileme Lagoon, "high" in the Oriental and Occidental Lagoons, and "low" in the Inferior Lagoon.
\end{abstract}

Keywords: Evaluation, distribution, shrimp, mangrove, coastal lagoons.

\footnotetext{
1 Universidad del Mar, campus Puerto Ángel

2 Instituto de Recursos e Industrias. Carretera a Zipolite, Ciudad Universitaria s/n, Distrito de San Pedro Pochutla A. P. 47 (70902 Puerto Ángel, Oaxaca, México).pch@angel.umar.mx*
}

Recibido 30-IV-2011

Aceptado 1-VIII-2012

DOI: http://dx.doi.org/10.15359/revmar.4.4 


\section{INTRODUCCIÓN}

Oaxaca es uno de los estados del sur de México con mayor diversidad biótica y riqueza cultural, cualidades que la hacen una región geográfica estratégica para el desarrollo local y nacional (Álvarez-López et al. 2002). Sin embargo, Oaxaca, Guerrero, Chiapas y Veracruz son los estados de México que presentan los mayores índices de marginación (Bozada-Robles, 2002).

El Sistema Lagunar Huave, localizado en el Istmo de Tehuantepec, Oaxaca, México, presenta una comunicación semipermanente con el Golfo de Tehuantepec (en el Océano Pacífico Tropical) a través de la "Boca de San Francisco". El sistema lagunar está formado de este a oeste por un conjunto de lagunas que son: Mar Tileme, Laguna Quirio, Laguna Superior, Laguna Inferior, Laguna Oriental y Laguna Occidental. Las lagunas Superior e Inferior están comunicadas permanentemente por la "Boca de Santa Teresa". El estero "Los Otates" comunica permanentemente a la Laguna Inferior con las lagunas Oriental y Occidental, estas últimas poseen pequeños canales de comunicación permanentes con el Golfo de Tehuantepec y son, respectivamente, los esteros Xhubabeza I y II (CONGEASA, 1993) (Fig. 1).

En las zonas Huave (Mar Tileme, Laguna Inferior, Laguna Oriental y Laguna Occidental) y Zapoteca (Laguna Superior) (Fig. 1), la pesca artesanal de escama y camarón se rige por el sistema de "Usos y Costumbres", con un alto sentido de la pertenencia étnica (Millán, 2003) y resistencia al impacto por la influencia entre comunidades e intromisiones externas (Espinoza-Tenorio et al. 2011). Por esta razón, desde tiempos prehispánicos, el Sistema Lagunar Huave ha estado dividido o "territorializado" en seis zonas culturales de pesca (Rodríguez y García, 1985) (Fig. 2). El sistema de "Usos y Costumbres" ha conferido autoridad, normas, procedimientos y formas de sanción al pescador indígena que acceda a las zonas culturales de pesca que no le correspondan (BozadaRobles, 2002); sin embargo, se reportan invasiones continuas a la zona cultural de

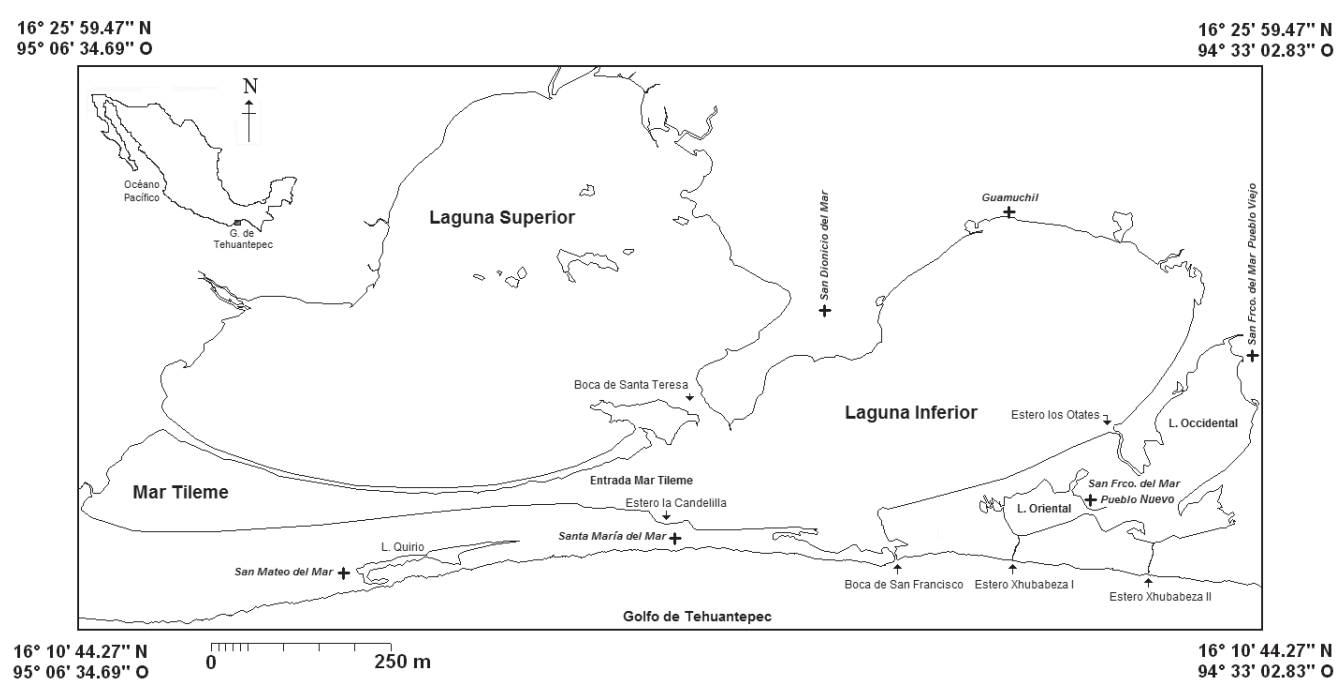

Fig. 1. Localización geográfica del Sistema Lagunar Huave

Fig. 1. Geographic location of the Huave Lagoon System 


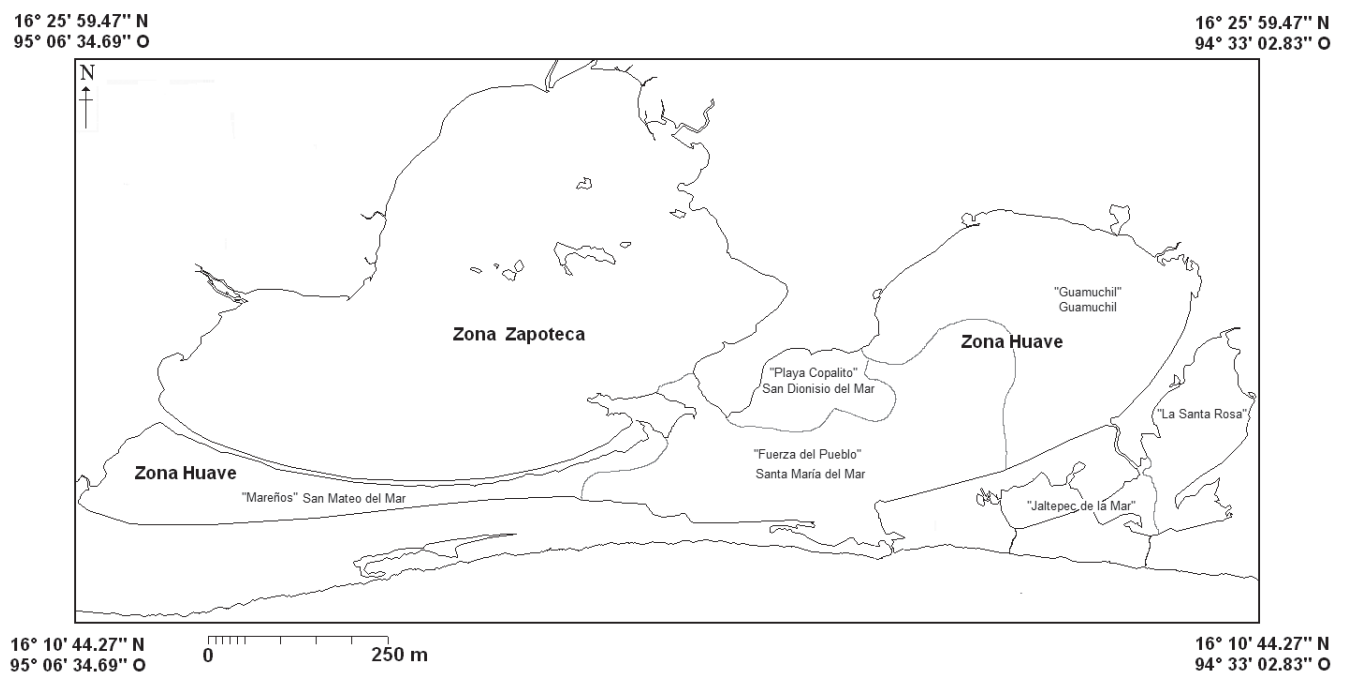

Fig. 2. Localización geográfica de las zonas culturales de pesca en el Sistema Lagunar Huave

Fig. 2. Geographic location of the cultural fishing zones in the Huave Lagoon System

pesca Zapoteca (Fig. 1) (Espinoza-Tenorio et al. 2011). El término huave o "la gente que se pudre en la humedad" fue acuñado por los zapotecas para señalar despectivamente a estos invasores. Pero los propios huaves se resisten a identificarse así, y en cambio, se autonombran Mero ikooc, que significa en huave, "verdaderos nosotros". La zona cultural de pesca Zapoteca no fue considerada en este trabajo.

Para la administración de la pesca artesanal en la zona Huave se han considerado históricamente aspectos socioeconómicos y culturales (Alcalá-Moya, 1999); a pesar de que estos son descritos a fondo por Bozada-Robles (2002), las sociedades cooperativas de producción pesquera huaves (SCPPH, en adelante) y el gobierno federal no han logrado consolidar un ordenamiento pesquero capaz de revertir el descenso de la captura de especies de interés comercial (principalmente camarón), problema que se agudiza, aún más, por los conflictos que desencadena el derecho étnico a utilizar los recursos pesqueros aún disponibles.
Los estudios realizados para las poblaciones de camarón del Golfo de Tehuantepec (Cervantes-Hernández et al. 2006; Ramos-Cruz et al. 2006; CervantesHernández, 2008; Cervantes-Hernández et al. 2010) son una alternativa viable para la estructuración de un plan regional de manejo marino-lagunar, ya que en estos se consideraron aspectos socioeconómicos, pesqueros y de la variación ambiental. Estos trabajos fueron parcialmente aceptados por las autoridades pesqueras del Estado de Oaxaca, debido a que no se incorporó información real (sino simulada) de los sistemas lagunares del Golfo de Tehuantepec (Huave, Mar Muerto, La Joya-Buenavista, Los Patos-Solo Dios, Carretas-Pereyra, Chantuto-Panzacola). A pesar de este gran avance para la pesca de camarón en este golfo, Cervantes-Hernández et al. (2006) señalaron que para estos sistemas lagunares persiste un vacío en la investigación pesquera; esta misma situación se visualiza en los estudios de la cobertura vegetal (principalmente mangle) y la variación ambiental e hidrológica. 
La "presión por pesca" ejercida por cada una de las SCPPH sobre el recurso camarón fue evaluada de 1999 al 2007. Además del esfuerzo de pesca $\left(\mathrm{f}_{\mathrm{i}}\right)$, la presión por pesca fue simulada con los siguientes parámetros: el nivel óptimo de captura $\left(\mathrm{C}_{\mathrm{OPT}}\right)$, el esfuerzo óptimo de pesca $\left(\mathrm{f}_{\text {OPT }}\right)$, la tasa de mortalidad natural $(\mathrm{M})$ y la tasa de explotación (E). La distribución de la captura artesanal fue explicada en función a la presión por pesca, la variación hidrológica de la temperatura superficial, la salinidad, la concentración de oxígeno disuelto y la turbidez (entre mayo y agosto-octubre de 2007), así como del estado actual de la cobertura de manglar de 2005 al 2007.

La generación de este tipo de información es relevante según Bozada-Robles (2002), en la estructuración de un ordenamiento pesquero que permita identificar y conciliar los problemas locales para el beneficio de todas las SCPPH, y lograr así el flujo de la información que se requiere para la unificación de un plan de manejo regional marino-lagunar.

\section{MATERIAL Y MÉTODOS}

Los datos utilizados en este trabajo fueron los registros mensuales de la captura artesanal de camarón $(\mathrm{kg})$ y del esfuerzo de pesca (días de pesca), período 19992007. Esta información fue proporcionada mediante hojas de arribo por las siguientes SCPPH: "Mareños" de San Mateo del Mar, "Fuerza del Pueblo" de Santa María del Mar, "Jaltepec de la Mar" de San Francisco del Mar "Pueblo Viejo", "La Santa Rosa" de San Francisco del Mar "Pueblo Nuevo", "Guamuchil" de Guamuchil y "Playa Copalito" de San Dionisio del Mar (Fig. 1).

Debido a que en el Sistema Lagunar Huave, la captura artesanal fue registrada sin la diferenciación entre especies comerciales, Farfantepenaeus californiensis (Holmes, 1900) “camarón café” y Litopenaeus vannamei (Boon, 1931) "camarón blanco", los análisis subsiguientes fueron realizados apegados a Cervantes-Hernández et al. (2006), considerando a ambas especies de camarón como un único recurso o una sola población distribuida en todo el sistema lagunar. Bajo estos supuestos y para cada una de las SCPPH, el nivel óptimo de captura y el esfuerzo óptimo de pesca fueron estimados según Schaefer (1991):

$$
\begin{aligned}
& \mathrm{C}_{\mathrm{OPT}}=-0.25 \cdot\left(\mathrm{a}^{2} / \mathrm{b}\right) \\
& \mathrm{f}_{\mathrm{OPT}}=-0.5 \cdot(\mathrm{a} / \mathrm{b})
\end{aligned}
$$

Donde a y b son la ordenada y la pendiente del modelo lineal: $\mathrm{CPUE}=\mathrm{a}+\left(\mathrm{b} \cdot \mathrm{f}_{\mathrm{i}}\right)$, CPUE es la captura por unidad de esfuerzo. $\mathrm{C}_{\text {OPT }}, \mathrm{f}_{\text {OPT }} \mathrm{y} \mathrm{f}_{\mathrm{i}}$ se definen como antes.

El valor mensual de la tasa de mortalidad total $(Z)$ se estimó con $\ln (\mathrm{CPUE})=$ a-(Z·n) (Berry, 1967), donde a es la ordenada al origen y n es el número de descensos que registró la CPUE. Los valores de $\mathrm{Z}$ obtenidos para cada $\mathrm{n}$ fueron asociados a los días de pesca promedio para estimar la tasa de mortalidad natural con $\mathrm{Z}=\mathrm{M}+$ $\left(\mathrm{q} \cdot \mathrm{fp}_{\mathrm{i}}\right)$ (Paloheimo, 1961), donde $\mathrm{q}$ es el coeficiente de capturabilidad (no utilizado en análisis subsiguientes) y $\mathrm{M}$ se define como antes. La tasa de explotación fue obtenida con $\mathrm{E}=\mathrm{F} / \mathrm{Z}$, donde $\mathrm{F}$ es la tasa de mortalidad por pesca obtenida con: $\mathrm{F}=$ M - Z (Ricker, 1975).

Con base en los parámetros $\mathrm{f}_{\mathrm{i}}, \mathrm{C}_{\mathrm{OPT}}$, $\mathrm{f}_{\text {OPT }}, \mathrm{M}$ y E, se diseñó un análisis multivariado de correspondencia (Hair et al. 1999) para simular la "presión por pesca" ejercida por cada una de las SCPPH sobre el recurso camarón. El diseño consideró los siguientes pasos: 
1. El valor esperado de los parámetros antes mencionados fue obtenido usando la siguiente relación: valor esperado de un $\mathrm{n}$ parámetro $=($ suma total de parámetros para una única SCPPH - la frecuencia del n parámetro en todas las SCPPH).

2. Los residuos obtenidos entre los parámetros observados y esperados fueron estandarizados a valores de similitud usando la distribución de $X_{i}^{2}$ con grados de libertad $=(\mathrm{i}-1) \cdot(\mathrm{j}-1)$. Donde i es el número de parámetros y j es el número de SCPPH analizadas.

3. La similitud representó a la presión por pesca, y los valores obtenidos fueron asignados a cada una de las SCPPH para visualizarlas en un gráfico perceptual. Usando el signo matemático del valor de la similitud, el eje $\mathrm{X}$ fue subdividido para identificar el efecto que tuvo la "presión por pesca"; de manera que, una "presión por pesca" a) muy baja y b) baja, se asignó a valores negativos de similitud; mientras que una "presión por pesca” c) media y d) alta, se asignó a valores positivos de similitud.

Una imagen del Sistema Lagunar Huave con proyección cartográfica equidistante, fue utilizada para insertar aleatoriamente en cada una de las zonas culturales de pesca (Fig. 2), el registro mensual de la captura artesanal correspondiente a cada una de las SCPPH; sin embargo, la información resultó ser insuficiente para cubrir el área total por zona, de manera que, los espacios vacíos fueron interpolados usando el análisis geoestadístico de "promedios móviles". Uriel (1995) señaló que un promedio móvil (D) de orden $w$ en el tiempo i es: $\mathrm{D}_{\mathrm{i}}=\left(\mathrm{C}_{1}+\mathrm{C}_{2}+\ldots+\mathrm{C}_{\mathrm{i}}\right) / \mathrm{w}$, donde $\mathrm{C}_{1}+\mathrm{C}_{2}+\ldots+\mathrm{C}_{\mathrm{i}}$ son los registros mensuales de la captura artesanal.
Un total de 108 imágenes mensuales de promedios móviles fueron obtenidas con el proceso anterior y con base en estas, un modelo geoestadístico de "regionalización" fue desarrollado usando la técnica de análisis de componentes principales de Hair et al. (1999). Con el proceso anterior fueron detectadas a lo largo del sistema lagunar, las áreas de mayor y menor persistencia de la captura artesanal. La distribución final de captura artesanal se obtuvo según Dallas (1999), usando la componente principal más significativa y que a manera de imagen, le fue sobrepuesta, la imagen regionalizada de la cobertura de manglar; esta última es tipo SPOT con proyección cartográfica equidistante, proporcionada por la Décimo Segunda Zona Naval de la Secretaría de Marina, México.

Según Hair et al. (1999), una componente principal es significativa si posee un autovalor mayor o igual a 1 , con un porcentaje de varianza cercano a 0.70 . Uriel (1995) definió la regionalización como:

$$
\mathrm{C}_{\mathrm{n}}=\mathrm{R}_{1} \mathrm{~V}_{1}+\mathrm{R}_{2} \mathrm{~V}_{2}+\ldots+\mathrm{R}_{\mathrm{i}} \mathrm{V}_{\mathrm{j}}
$$

Donde:

$\mathrm{C}_{\mathrm{n}}$, es una de las $\mathrm{n}$ imágenes regionalizadas generadas por el ACP.

$\mathrm{R}_{\mathrm{i}}$, son las imágenes mensuales de promedios móviles.

$\mathrm{V}_{\mathrm{j}}$, son los autovalores asociados a cada $R_{\mathrm{i}}$.

Las condiciones hidrológicas superficiales del Sistema Lagunar Huave fueron descritas en función de la temperatura superficial $\left({ }^{\circ} \mathrm{C}\right)$, la salinidad (UPS), la concentración de oxígeno disuelto (mg/l) y la turbidez (UTN). El registro de estas variables hidrológicas se realizó in situ a 0.20 $\mathrm{m}$ de profundidad, en 33 estaciones georreferidas, distribuidas sistemáticamente 
en el Mar Tileme, la Laguna Inferior y las lagunas Occidental y Oriental (Fig. 3). El registro de cada una de las variables hidrológicas fue obtenido con una sonda multivariable 6600 de YSI $^{\circledR}$ (Yellow Spring Inc., acoplada a una consola 650 MDS YSI ${ }^{\circledR}$ ). Para realizar la campaña de recolecta de las variables hidrológicas, se utilizó una lancha de fibra de vidrio con 23 pies de eslora y motor YAMAHA de 60 Hp. Los períodos de recolecta fueron del 11 al 13 de mayo (temporada de estiaje) y del 14 de agosto al 12 de octubre (temporada de lluvias) de 2007. La información así obtenida fue interpolada fusionando ambos períodos de recolecta para obtener una tendencia final.

El programa ILWIS 3.0 (ILWIS, 2007) se utilizó para ejecutar los modelos geoestadísticos.

\section{RESULTADOS}

Los parámetros obtenidos para cada una de las SCPPH de 1999 al 2007 se muestran en el cuadro 1. A través de la captura óptima, se observó que en "Jaltepec de la Mar" y "La Santa Rosa" (en las lagunas Oriental y Occidental), la extracción fue de $54025 \mathrm{~kg}(96.58 \%$ del total de la captura artesanal); el resto, $3.42 \%$, estuvo integrado de la siguiente manera: "Mareños" con $0.35 \%$ (en el Mar Tileme), "Fuerza del Pueblo" con $0.85 \%$ (entrada al Mar Tileme) y "Guamuchil" con $2.21 \%$ (secciones norte y media de la Laguna Inferior).

La tasa de mortalidad natural en el Sistema Lagunar Huave osciló entre 0.31 y 0.82 mensual. Los valores más altos fueron obtenidos para "Mareños" $(\mathrm{M}=0.82$ mensual), "Fuerza del Pueblo" $(\mathrm{M}=0.65$ mensual) y "Guamuchil" ( $\mathrm{M}=0.52$ mensual); estos valores fueron contrastantes con los estimados en "Jaltepec de la Mar" ( $\mathrm{M}=0.34$ mensual) y "La Santa Rosa" (M $=0.31$ mensual) (Cuadro 1). En estas últimas, la tasa de explotación resultó mayor en comparación con el resto de las $\mathrm{SCPPH}$, donde la tasa de mortalidad natural fue mayor a la tasa de explotación (Cuadro 1).

La presión por pesca se describe en la figura 4. Estos resultados sugieren,

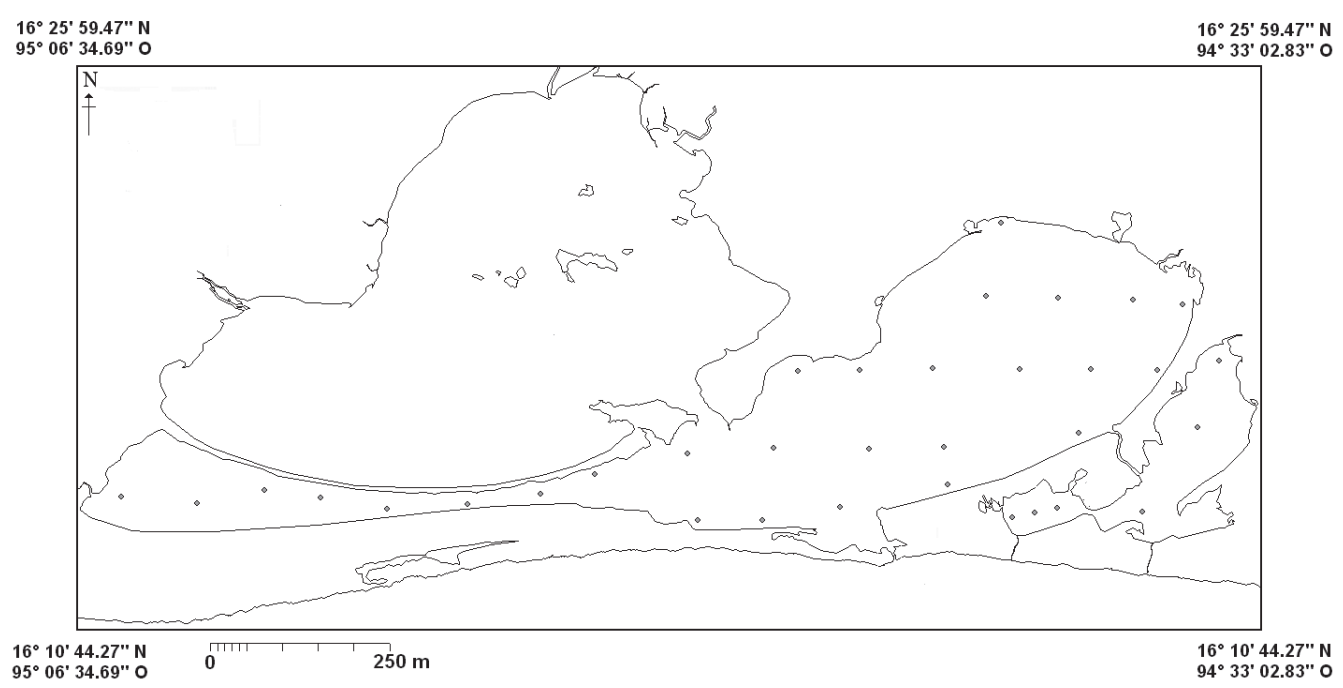

Fig. 3. Localización geográfica de las estaciones de muestreo hidrológicas en el Sistema Lagunar Huave

Fig. 3. Geographic location of the hydrological monitoring stations in the Huave Lagoon System 
Cuadro 1. Parámetros estimados para cada una de las SCPPH entre 1999 y 2007. Nivel óptimo de captura $\left(\mathrm{C}_{\mathrm{OPT}}\right)$, esfuerzo óptimo de pesca $\left(\mathrm{f}_{\mathrm{OPT}}\right)$, esfuerzo de pesca $\left(\mathrm{f}_{\mathrm{i}}\right)$, tasa de explotación (E), tasa de mortalidad natural $(\mathrm{M})$, parámetro no estimado (p.n.e.)

Table 1. Estimated parameters for each SCFPH between 1999 and 2007. Maximum sustainable yield $\left(\mathrm{C}_{\mathrm{OPT}}\right)$, level of fishing effort at which the maximum sustainable yield is achieved $\left(\mathrm{f}_{\mathrm{OPT}}\right)$, fishing effort $\left(\mathrm{f}_{\mathrm{i}}\right)$, exploitation rate $(\mathrm{E})$, natural mortality rate $(\mathrm{M})$, unestimated parameter (p.n.e. Acronym in Spanish)

\begin{tabular}{lccccc}
\hline SCPPH & $\begin{array}{c}\mathbf{C}_{\mathbf{O P T}} \\
\mathbf{k g}\end{array}$ & $\begin{array}{c}\mathbf{f}_{\text {OPT }} \\
\text { dias de pesca }\end{array}$ & $\begin{array}{c}\mathbf{E} \\
\text { mensual }\end{array}$ & $\begin{array}{c}\mathbf{M} \\
\text { mensual }\end{array}$ & $\begin{array}{c}\mathbf{f}_{\mathbf{i}} \\
\text { días de pesca }\end{array}$ \\
\hline Mareños & 200 & 29 & 0.12 & 0.82 & 28 \\
Fuerza del Pueblo & 477 & 19 & 0.38 & 0.65 & 28 \\
Jaltepec de la Mar & 24247 & 45 & 0.75 & 0.34 & 30 \\
La Santa Rosa & 29778 & 58 & 0.91 & 0.31 & 28 \\
Guamuchil & 1239 & 56 & p.n.e. & 0.52 & 29 \\
Playa Copalito & p.n.e. & p.n.e. & p.n.e. & p.n.e. & 27 \\
\hline
\end{tabular}

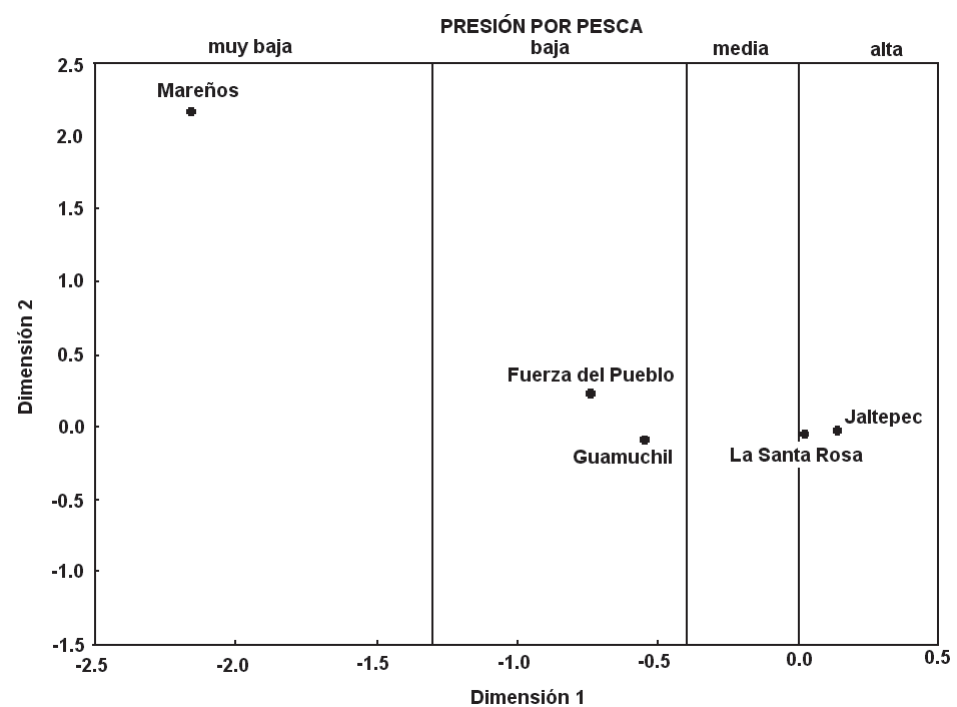

Fig. 4. Modelo de presión por pesca sobre el recurso camarón en el Sistema Lagunar Huave, período 1999-2007

Fig. 4. Fishing pressure model on shrimp resources in the Huave Lagoon System, 1999-2007 period

consistentemente con el cuadro 1, que "Jaltepec de la Mar" y "La Santa Rosa" ejercieron una alta presión por pesca sobre el recurso camarón en las lagunas Oriental y Occidental; el extremo opuesto fue "Mareños", con una muy baja presión por pesca en el Mar Tileme; mientras que, "Guamuchil" y "Fuerza del Pueblo" evidenciaron una baja presión por pesca.
Ninguna SCPPH mostró una presión por pesca media y para el caso particular de "Playa Copalito", esta no pudo ser estimada, razón por la que esta SCPPH no estuvo inscrita en la figura 4.

En la figura 5 se muestra la distribución de la captura artesanal obtenida para el Sistema Lagunar Huave. Esta componente principal generó un autovalor 
de 2.4 con $75 \%$ de varianza $(P<0.05)$. El resto de las componentes principales (107) fueron descartados por explicar menos del 50\% $(P>0.05)$.

La distribución de la captura artesanal mostró valores altos en las lagunas Oriental y Occidental, la zona cultural de pesca que menos captura artesanal registró fue el Mar Tileme. Un gradiente descendente de la captura artesanal, fue observado desde las lagunas Oriental y Occidental, con dirección hacia la costa norte de la Laguna Inferior, desvaneciéndose hacia la entrada del Mar Tileme (Fig. 5). Todas las SCPPH invirtieron aproximadamente el mismo esfuerzo de pesca (Cuadro 1), pero la captura artesanal obtenida no resultó equitativa entre las diferentes zonas culturales de pesca (Cuadro 1 y Fig. 5).

Una extensa cobertura de manglar fue visualizada alrededor de las lagunas Oriental y Occidental. Esta cobertura fue menos densa y fraccionada entre el estero "La Candelilla" y la "Boca de San Francisco"; el resto del Sistema Lagunar Huave evidenció pequeños parches aleatorios de manglar en su periferia (Fig. 5).

Las lagunas Oriental y Occidental mostraron gradientes de variación bien definidos en las variables hidrológicas (27.0$28.6^{\circ} \mathrm{C}, 11.1-22.5$ UPS, $6.7-7.6 \mathrm{mg} / 1$ y 1.6-84 UTN) (Fig. 6). Estas resultaron homogéneas en el Mar Tileme, con valores extremos $\left(29.2-29.7^{\circ} \mathrm{C}, 44.0-54.7\right.$ UPS, $5.0-6.7 \mathrm{mg} / \mathrm{l}$ y $1.6 \mathrm{UTN})$, en comparación con el resto del sistema lagunar (Fig. 6). Valores intermedios en las variables hidrológicas (excepto la turbidez) fueron observados con un gradiente descendente desde las lagunas Oriental y Occidental, hacia la costa norte de la Laguna Inferior, desvaneciéndose en las inmediaciones del Mar Tileme (Fig. 6).

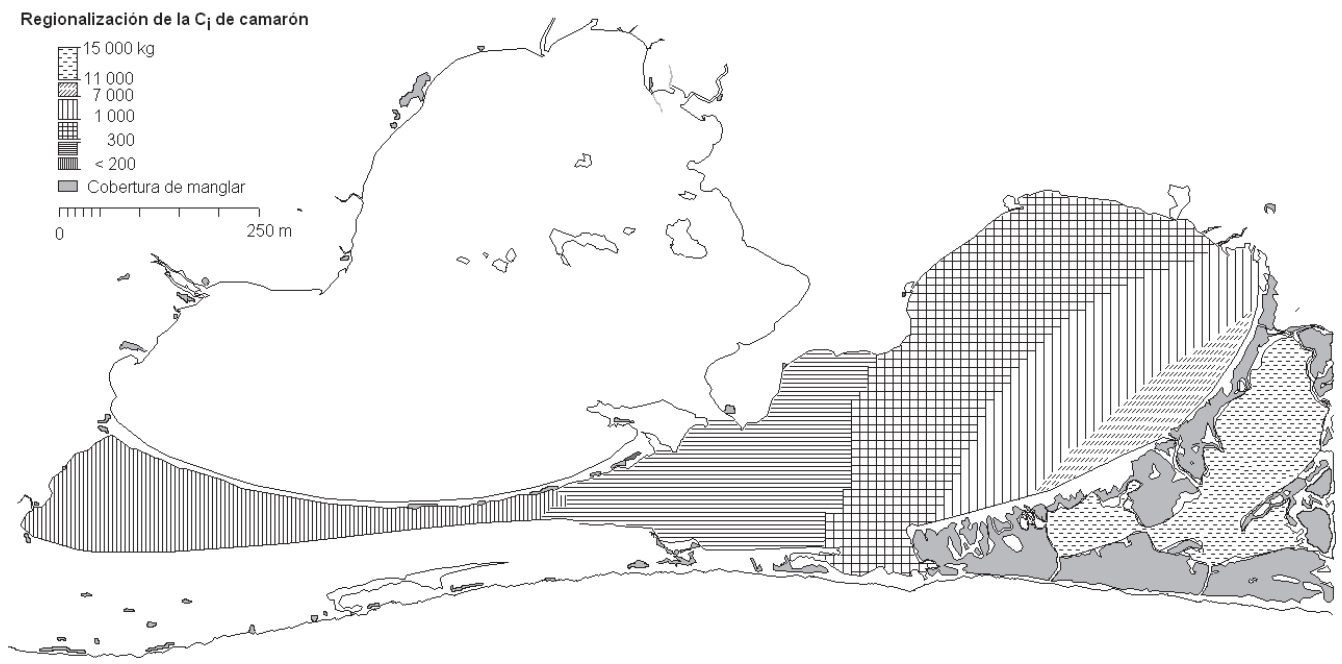

Fig. 5. Modelo de distribución para la captura artesanal (de 1999 al 2007) y la cobertura de manglar (entre 2005 y 2007) en el Sistema Lagunar Huave

Fig. 5. Artisanal shrimp catch distribution model (from 1999 to 2007) and mangrove coverage (between 2005 and 2007) in the Huave Lagoon System 


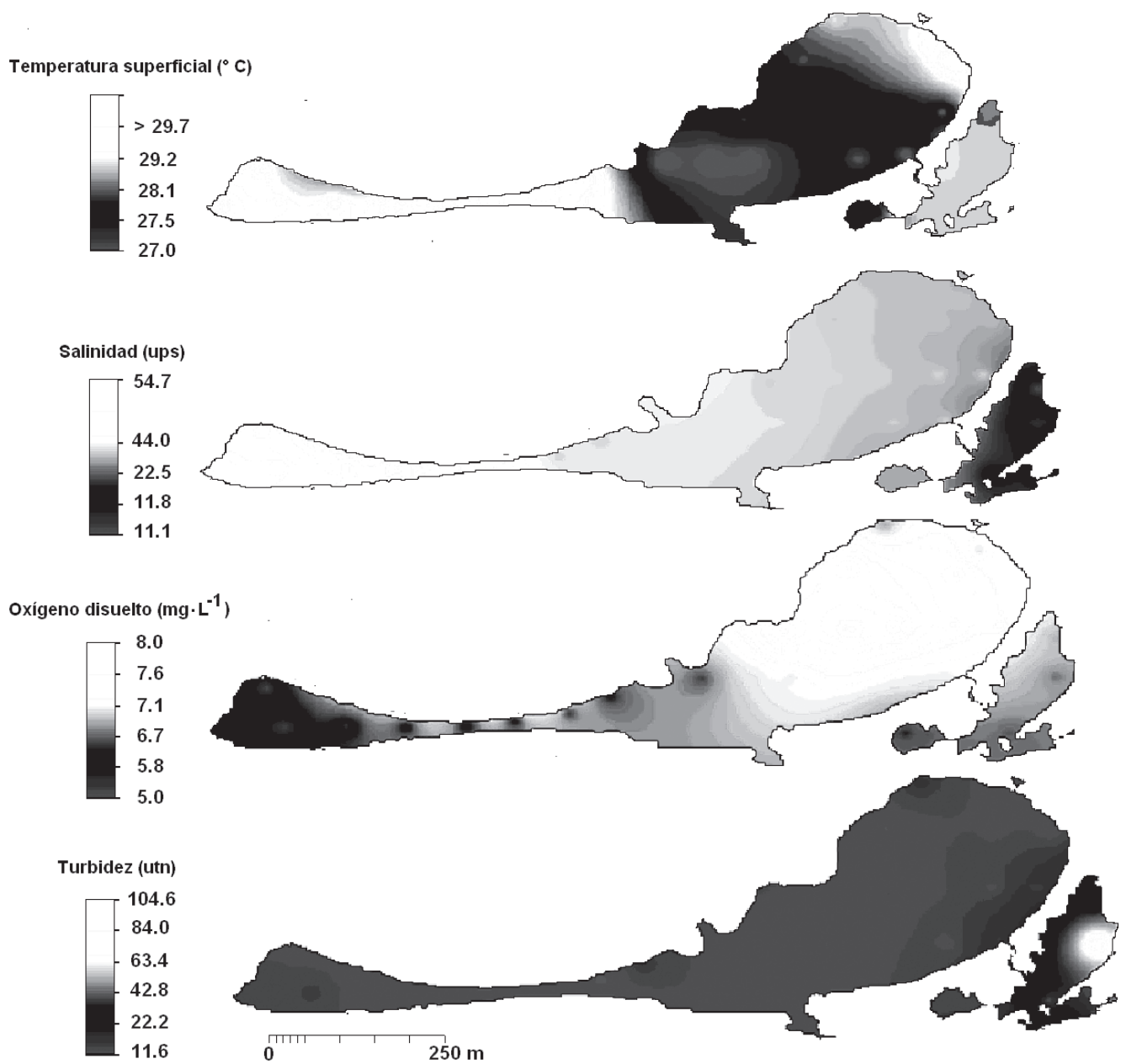

Fig. 6. Modelo de promedios móviles para cada una de las variables hidrológicas en el Sistema Lagunar Huave. Para este caso fueron consideradas las temporadas de muestreo de mayo (temporada de estiaje) y de agosto a octubre de 2007 (temporada de lluvias)

Fig. 6. Moving average model for each hydrological variable in the Huave Lagoon System. In this case, the sample seasons of May (drought season) and from August to October 2007 (rainy season) were considered

\section{DISCUSIÓN}

Para desarrollar el modelo de Schaefer en cualesquiera de sus versiones, es necesario cumplir con el supuesto de que la pesquería se encuentra en equilibrio o no (Hilborn y Walters, 1992; Punt y Hilborn, 1996). Debido a la aparente violación de estos supuestos y a la simplicidad del modelo, Maunder (2003) mencionó que este debería ser descartado como herramienta de evaluación pesquera. Sin embargo, Williams y Prager (2002) replantearon los supuestos e indicaron que las diferentes versiones del modelo de Schaefer consideran, conjuntamente, el estado de equilibrio y no equilibrio, por lo que resulta innecesario corroborar estos 
supuestos antes de su aplicación. ChienHsiung (2004) confirmó lo antes expuesto e indicó que este modelo es una de las herramientas más poderosas en el análisis y el manejo de las pesquerías; sin embargo, al igual que otros modelos pesqueros, es sensible a la unidad del esfuerzo pesquero que se utilice, ya sea estandarizado o no.

Independientemente del estado de equilibrio en el cual podría encontrarse la pesca artesanal de camarón en el Sistema Lagunar Huave, no se utilizó un modelo dinámico de biomasa, ya que la captura artesanal estuvo integrada por las dos especies de camarón de interés comercial, y que de acuerdo con Cervantes-Hernández et al. (2006) resulta ilógico asignar a ambas especies (consideradas como una sola población) un valor único de los parámetros dinámicos: tasa intrínseca de crecimiento poblacional (r), tamaño máximo de población (k), capturabilidad (q) y biomasa inicial (Bo).

El ciclo de vida de los camarones de la familia Penaeidae es de aproximadamente 16 meses y se desarrolla entre los ambientes marino y lagunar (Gracia et al. 1997). En el Golfo de Tehuantepec (zona 90 de pesca), Cervantes-Hernández (2008) identificó los períodos de reclutamiento marino y lagunar para $F$. californiensis de 1989 a 1998; en este trabajo, el "reclutamiento marino" fue definido como el movimiento natural de camarones juveniles desde el interior de los sistemas lagunares de Oaxaca (Huave y Mar Muerto) y Chiapas (La Joya-Buenavista, Los Patos-Solo Dios, Carretas-Pereyra y Chantuto-Panzacola) hacia la zona de pesca 90, y el "reclutamiento lagunar", como el movimiento de poslarvas de camarón desde la zona de pesca 90 hacia el interior de estos sistemas lagunares.

El exceso en la captura artesanal en los sistemas lagunares del Golfo de
Tehuantepec fue identificado por Cervantes-Hernández et al. (2006) como la principal causa de una relación inversa entre la pesca artesanal y la captura marina de camarón $(\mathrm{R}=-0.5377, P<0.05)$. Estos autores identificaron al Sistema Lagunar Huave como uno de los sistemas lagunares con menor flujo de reclutamiento marino hacia la zona de pesca 90, superado en producción camaronera por el resto de los sistemas lagunares (Fig. 7).

Utilizando nuevas técnicas de simulación pesquera, se refutó la idea de que el descenso en el reclutamiento marino proveniente del Sistema Lagunar Huave haya sido ocasionado exclusivamente por un exceso en la captura artesanal. En complemento a lo señalado por Cervantes-Hernández et al. (2006), el presente trabajo evidenció graves problemas de deterioro ambiental, que según las hojas de arribo proporcionadas por las SCPPH y lo señalado por estos últimos autores, esta situación se ha venido desarrollando desde la década de los ochenta. La situación ambiental observada en el Sistema Lagunar Huave deberá ser considerada en trabajos futuros, si se pretende comprender el nuevo rumbo que está tomando la pesca artesanal y marina de camarón en el Golfo de Tehuantepec.

El Mar Tileme resultó hiperhalino (4454.7 UPS), debido a las altas temperaturas registradas $\left(29.2-30^{\circ} \mathrm{C}\right)$, con bajos niveles de oxígeno disuelto (5.0-6.7 mg/1). Gracia y Soto (1990) reportaron que a 34 UPS, se observó una reducción dramática de la captura artesanal de camarón en la Laguna de Términos, Campeche, México. Rosas et al. (1999) indicaron que en el intervalo de 15 a 35 UPS y bajo condiciones de hipoxia, Litopenaeus setiferus (Linnaeus, 1767) modifica su metabolismo para mantener la actividad biológica, reduciendo hasta en un 


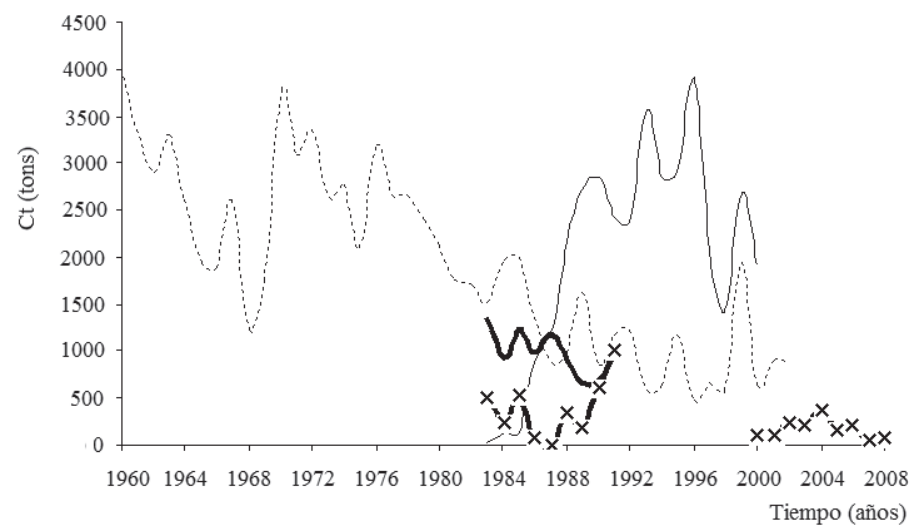

Fig. 7. Registros históricos para la captura marina de camarón del Golfo de Tehuantepec (línea negra discontinua), captura ribereña de camarón del Estado de Chiapas (línea negra continua delgada), captura ribereña de camarón del Mar Muerto (línea negra continua gruesa) y captura ribereña de camarón del SLH (línea negra discontinua con símbolo (x)

Fig. 7. Historical records for shrimp marine catches from the Gulf of Tehuantepec (black dashed line), artisanal shrimp catches from the State of Chiapas (thin continuous black line), artisanal shrimp catches from the Mar Muerto Lagoon (thick continuous black line) and artisanal shrimp catches from Huave Lagoon System (discontinuous black line with symbol (x)

65\% el consumo de oxígeno; sin embargo, esta situación disminuye al incrementar la salinidad, debido a su limitada capacidad para depositar sustancias metabólicas de reserva en los tejidos, que se agudiza según Sánchez et al. (1991) por los altos requerimientos de oxígeno para mantener la actividad muscular.

A pesar de que el oxígeno disuelto en el Mar Tileme resultó en una menor concentración en relación con el resto del Sistema Lagunar Huave, este no resultó perjudicial fisiológicamente para L. vannamei y $F$. $c a$ liforniensis. Lo anterior está sustentado en el trabajo de Fotheringham y Weissberg (1979), quienes indicaron que estas especies sufren de estrés y luego la muerte, bajo una exposición prolongada de hipoxia entre 1.49 y $1.9 \mathrm{mg} / 1$, respectivamente.

En el Mar Tileme, el efecto de las variables hidrológicas sobre la distribución de la captura artesanal estuvo reflejado, en parte, en el valor de la tasa de mortalidad natural estimada $(\mathrm{M}=0.82$ mensual). Este valor superó las estimaciones reportadas para las especies marinas de camarón del Golfo de Tehuantepec, que según RamosCruz et al. (2006) son de $\mathrm{M}=0.22$ mensual para $F$. californiensis y $\mathrm{M}=0.20$ mensual para L. vannamei, períodos 1989-1998 y 1992-1998, respectivamente.

Los camarones del género Penaeus son organismos estuarinos dependientes y durante la fase de poslarva ingresan a los estuarios y lagunas costeras para su desarrollo y protección (Baxter y Renfro, 1967); esta última, según Gracia et al. (1997), depende en gran medida de la presencia y conservación de la cobertura vegetal.

Para Farfantepenaeus duorarum (Burkenroad, 1939) del Banco de Campeche, México, Cervantes-Hernández y Gracia (2011) reportaron un incremento gradual en el valor de la tasa de mortalidad natural, entre 1974 y 1989, debido a un incremento en la deforestación ocasionada 
por el aumento de las actividades petroleras en las zonas de pesca marina y lagunar.

Martínez-Ojeda (1993) reportó extensas áreas de manglar (Rhizophora mangle, Laguncularia racemosa y Conocarpus erectus alternando con el epazotillo Batis maritima) al oeste del Mar Tileme, específicamente en los sitios planos y arenosos con vista al Golfo de Tehuantepec. En la actualidad y para esta última área, la cobertura de manglar se ha reducido a pequeños parches aleatorios localizados entre el estero "La Candelilla" y la "Boca de San Francisco"; el resto del Sistema Lagunar Huave evidenció una alta deforestación de manglar, excepto en las inmediaciones de las lagunas Oriental y Occidental.

A pesar de que las comunidades huaves han mostrado un fuerte arraigo cultural sobre el uso de la madera de mangle en diversos procesos productivos (EspinozaTenorio et al. 2011), la construcción de la presa "Presidente Benito Juárez" en 1961 ha contribuido en forma gradual a la deforestación, debido a la modificación de los diferentes afluentes fluviales, que históricamente desembocaron al oeste del Mar Tileme y que en la actualidad son utilizados en el Distrito de Riego 19 para beneficiar a los municipios de San Blas Atempa, San Pedro Huilotepec, Santa María Xadani y Juchitán de Zaragoza (EspinozaTenorio et al. 2011).

En las lagunas Oriental y Occidental, la captura artesanal de camarón fue abundante bajo condiciones óptimas de cobertura de manglar y calidad hidrológica, estas, a su vez, redujeron la tasa de mortalidad natural, permitiendo el incremento de la presión por pesca en "Jaltepec de la Mar" y "La Santa Rosa". Al respecto, Ramos-Cruz (2000) reportó que la captura artesanal extraída de estas lagunas ha fluctuado entre 62.04 y $65.89 \mathrm{~kg} / \mathrm{ha} / \mathrm{año}$, superando a las reportadas para el Mar Tileme, entre 13.14 y $2.01 \mathrm{~kg} / \mathrm{ha} / \mathrm{año}$. La presencia de los canales de comunicación o esteros Xhubabeza I y II (Fig. 1) confiere a las lagunas Occidental y Oriental un medio permanente de intercambio hidrológico con el Golfo de Tehuantepec, independizándose así de los períodos de "Boca Abierta", que condiciona la "Boca de San Francisco" para incrementar la magnitud de la captura artesanal en todo el Sistema Lagunar Huave.

Una baja "presión por pesca" se registró en las SCPPH "Fuerza del Pueblo" y "Guamuchil"; pero de acuerdo con los resultados obtenidos, la captura artesanal extraída a lo largo de la Laguna Inferior (Fig. 1) no pudo generarse dentro de esta, por lo que suponemos que todo el volumen capturado fue importado a través del estero "Los Otates", debido al exceso de producción generado en las lagunas Oriental y Occidental.

La SCPPH "Playa Copalito" presentó muy pocos registros de arribo para camarón; por tal motivo ninguno de los parámetros de interés pudo ser estimado; sin embargo, esta SCPPH posee un extenso registro para la captura de escama proveniente íntegramente de la zona Zapoteca.

Es necesario mencionar que los episodios de apertura y cierre de la "Boca de San Francisco" no han sido documentados; sin embargo, los pescadores de las SCPPH indicaron que esta estuvo cerrada de mayo de 2006 a septiembre de 2010. Durante este período denominado "Boca Cerrada", fueron obtenidos los registros de las variables hidrológicas (mayo y agosto-octubre de 2007); mientras que, la simulación pesquera cubrió 7.4 años en "Boca Abierta" (de 1999 a abril de 2005) y 1.8 años en "Boca Cerrada" (de mayo de 2005 al 2007). La presión por pesca, la distribución de la captura artesanal y 
la cobertura de manglar fueron utilizadas como productos de tendencia final para poder asociarse con los resultados hidrológicos y describir, así, resultados bajo el predominio del período "Boca Cerrada".

Es recomendable ampliar este tipo de estudios al resto de los sistemas lagunares del Golfo de Tehuantepec para identificar y comprender las fallas naturales en el reclutamiento marino. En el caso particular del Sistema Lagunar Huave, será necesario monitorear el recurso camarón en el actual período de "Boca Abierta", integrando la información que resulte de escama y camarón en la zona Zapoteca. Como dato adicional, la "Boca de San Francisco" ha permanecido abierta desde septiembre de 2010 hasta la fecha.

\section{AGRADECIMIENTOS}

Este trabajo fue desarrollado en la Universidad del Mar, Puerto Ángel, Oaxaca, con el apoyo económico del convenio de colaboración Plan de Manejo Acuícola y Pesquero de la Zona Huave, SDGCONAPESCA-FAPC-UMAR. Agradecemos a la Décimo Segunda Zona Naval, representada por el Comandante José María Ortegón Cisneros y a la Tte. de Navío Luz María Salas Flores, por la imagen regionalizada de la cobertura de manglar, así como a los revisores anónimos por las sugerencias y comentarios.

\section{BIBLIOGRAFÍA}

Alcalá-Moya, G. (1999). Con el agua hasta los aparejos: pescadores y pesquerías en el Soconusco, Chiapas. México, D. F.: CIESAS/UNICACH/CIAD. Álvarez-López, B., Morales-Pacheco, O. \& Cruz-Hernández, J. A. (2002, octubre). La pesca artesanal en Oaxaca.
Ponencia presentada en el Primer Foro Científico de la Pesca Artesanal, Guaymas, Sonora, México.

Baxter, K. N. \& Renfro, W. C. (1967). Seasonal occurrence and size distribution of postlarval brown and white shrimp near Galveston, Texas, with notes on species identification. Fish. Bull., 66(1), 149-158.

Berry, R. J. (1967). Dynamic of the Tortugas (Florida) pink shrimp population. $\mathrm{Ph} . \mathrm{D}$. thesis was not published, University of Rhode Island, EE.UU.

Bozada-Robles, R. L. (2002). Los pescadores zapotecas del complejo lagunar del istmo de Tehuantepec, Oaxaca, México. Recuperado en febrero 12, 2011. Disponible en http://www.ciesas-golfo.edu.mx/istmo/docs/pescadoreszapotecas/indice.htm

Cervantes-Hernández, P., Ramos-Cruz, S. \& Gracia, A. (2006). Evaluación del estado de la pesquería de camarón en el Golfo de Tehuantepec. Hidrobiol., 16(3), 233-239.

Cervantes-Hernández, P. (2008). Method to obtain abundance indices in the population of Farfantepenaeus californiensis (Holmes, 1900) from the Gulf of Tehuantepec, Oaxaca, México. Rev. Biol. Mar. Oceanogr., 43(1), 111-119.

Cervantes-Hernández, P., Flores-Gómez, A., Serrano-Guzmán, S. J., RamosCruz, S. \& Gallardo-Berumen, M. I. (2010). Historical exploitation and evaluation of brown shrimp fishery Farfantepenaeus californiensis (Decapoda, Dendrobranchiata) in the Gulf of Tehuantepec, Oaxaca, México. PANAMJAS, 5(4), 486-494.

Cervantes-Hernández, P. \& Gracia, A. (2011). Análisis de la mortalidad para el camarón rosado Farfantepenaeus duorarum (Decapoda, Dendrobranchiata) 
del Banco de Campeche, México. PANAMJAS, 6(2), 100-108.

Chien-Hsiung, W. (2004, August). Improvement of the Schaefer model and its application. Conference. $17^{\text {th }}$ Meeting of the Standing Committee on Tuna and Billfish. Majuro, Marshall Islands. CONGEASA. (1993). Caracterización biológica pesquera del camarón en la Laguna Oriental, Occidental, Inferior $y$ marismas del Estado de Oaxaca. Oaxaca, México: Sociedad Cooperativa de Producción Pesquera Jaltepec de la Mar, Salina Cruz, Oaxaca, México.

Dallas, E. J. (1999). Métodos multivariados aplicados al análisis de datos. Kansas, EE.UU.: Thomson Paraninfo, S.A.

Espinoza-Tenorio, A., Bravo-Peña, L. C., Serrano-Guzmán, J. S., Ronsón-Paulín, J. A., Ahumada, M. A., Cervantes-Hernández, P., Robles-Zavala, E., Fuentes, M. P. \& Gallardo-Berumen, M. I. (2011). La diversidad étnica como factor de planeación pesquera artesanal: Chontales, Huaves y Zapotecas del Istmo de Tehuantepec, Oaxaca, México. En G. Alcalá \& A. Camargo (Eds.), Pescadores en América Latina y el Caribe: espacio, población, producción y política, vol. I (pp. 167-216). México, D. F.: Facultad de Ciencias, Unidad Multidisciplinaria de Docencia e Investigación - SISAL, Universidad Nacional Autónoma de México.

Fotheringham, N. \& Weissberg, G. H. (1979). Some causes, consequences and potential environmental impacts of oxygen depletion in the northern Gulf of Mexico. Proc. 11th Annu. Offshore Tech. Conf., 4(3611), 2205-2208.

Gracia, A. \& Soto, L. (1990). Populations study of the penaeid shrimp of Terminos lagoon, Campeche, Mexico. An. Inst. Cienc. Mar Limnol., 17(2), 241-255.
Gracia, A., Vázquez-Bader, A. R., Arreguín-Sánchez, F., Schultz-Ruiz, L. E. \& Sánchez, J. A. (1997). Ecología de camarones peneidos. En D. FloresHérnandez, P. Sánchez-Gil, J. C. Seijo \& F. Arreguín-Sánchez (Eds.), Análisis $y$ diagnóstico de los recursos pesqueros críticos del Golfo de México (pp. 127-144). Campeche, México: Centro de Ecología, Pesquerías y Oceanografía del Golfo de México.

Hair, J. F., Anderson, R. E., Tatham, R. L. \& Black, W. C. (1999). Multivariate data analysis. Iberia, Madrid: Prentice Hall.

Hilborn, R. \& Walters, C. J. (1992). Quantitative Fisheries Stock Assessment: Choice, Dynamics and Uncertainty. London, Inglaterra: Chapman \& Hall.

Ilwis (Integrated Land and Water Information System) version 3.0. (2007). International Institute for Geo-Information Science and Earth Observation, GNU licence. Took at June 01, 2008. Available in http://ilwis.wikispaces.com/home.

Martínez-Ojeda, O. (1993). El medio biofisico del Istmo de Tehuantepec: una revisión. Recuperado en marzo 28, 2011. Disponible en http://www.ciesasgolfo.edu. $\mathrm{mx} /$ istmo/docs/tesis/LAS\%20PESQUERIAS\%20DEL\%20COMPLEJO\%20 LAGUNAR/ CAPITULO\%20II.pdf.

Maunder, M. N. (2003). Is it time to discard the Schaefer model from the stock assessment scientist's toolbox? Fish. Res., 61, 145-149.

Millán, S. (2003). Huaves. En C. EnriqueSerrano \& L. Cruz-González (Eds.), Pueblos indígenas del México contemporáneo. México, D. F.: CDI/PNUD.

Paloheimo, J. (1961). Studies on estimation of mortalities, comparison of a method described by Beverton and Holt and a new linear formula. J. Fish. Res. Bd. Can., 18(5), 645-662. 
Punt, A. E. \& Hilborn, R. (1996). Biomass dynamic models: User's Manual. Computarized Information Series (Fisheries) No. 10. Rome, Italy: FAO.

Ramos-Cruz, S. (2000). Composición por tallas, edad y crecimiento de Litopenaeus vannamei (Natantia: Penaeidae), en la Laguna Mar Muerto, Oaxaca-Chiapas, México. Rev. Biol. Trop., 48(4), 873-882.

Ramos-Cruz, S., Sánchez-Meraz, B., Carrasco-Ayuso, F. \& Cervantes-Hernández, P. (2006). Estimación de la tasa de mortalidad natural de Farfantepenaeus californiensis (Holmes, 1900) y Litopenaeus vannamei (Boone, 1931), en la zona costera del Golfo de Tehuantepec, México. Rev. Biol. Mar. Oceanog., 41(2), 221-229.

Ricker, W. E. (1975). Computation and interpretation of biological statistics of fish populations. Bull. Fish. Res. Board Can., 191, 1-382.

Rodríguez, R. \& García, I. (1985). Los pescadores de Oaxaca y Guerrero. México, D. F.: SEP.
Rosas, C., Martínez, E., Gaxiola, G., Brito, R., Sánchez, A. \& Soto, L. A. (1999). The effect of dissolved oxygen and salinity on oxygen consumption, ammonia excretion and osmotic pressure of $\mathrm{Pe}$ naeus setiferus (Linnaeus) juveniles. $J$. Exp. Mar. Biol. Ecol., 234, 41-57.

Sánchez, A., Rosas, C., Escobar, E. \& Soto, L. A. (1991). Skeleton weight free oxygen consumption related to adaptations to environment and habits of six crustacean species. Comp. Biochem. Physiol., 100A, 69-73.

Schaefer, M. B. (1991). Some aspects of the dynamics of populations important to the management of the commercial marine fisheries. Bull. Mat. Biol., 53(1/2), 253-279.

Uriel, E. (1995). Análisis de datos, series temporales y análisis multivariante. Madrid, España: Alfa Centauro, S. A.

Williams, E. H. \& Prager, M. H. (2002). Comparison of equilibrium and nonequilibrium estimators for the generalized production model. Can. J. Fish. Aquat. Sci., 59(9), 1533-1552. 